\title{
Physical exercise as an add-on treatment to cognitive behavioural therapy for anxiety: a systematic review
}

\author{
Kristian Pihl Frederiksen ${ }^{1, \star}$, Silje Haukenes Stavestrand ${ }^{2,3}$ (D), Simen Kildahl Venemyr ${ }^{4}$, \\ Kristine Sirevåg ${ }^{2,3}$ and Anders Hovland ${ }^{2,3}$ \\ ${ }^{1}$ Diakonhjemmet Hospital, Oslo, Norway, ${ }^{2}$ Solli District Psychiatric Centre (DPS), Nesttun, Norway, ${ }^{3}$ Department of Clinical \\ Psychology, University of Bergen, Bergen, Norway and ${ }^{4}$ Bærum District Psychiatric Centre (DPS), Gjettum, Norway \\ ${ }^{*}$ Corresponding author. Email: kristian.pihl.frederiksen@gmail.com
}

(Received 29 June 2020; revised 23 January 2021; accepted 28 January 2021; first published online 08 March 2021)

\begin{abstract}
Background: Cognitive behavioural therapy (CBT) is currently the treatment of choice for most anxiety disorders. Yet, with recovery rates of approximately $50 \%$, many patients fail to achieve complete remission. This has led to increased efforts to enhance treatment efficacy. Physical exercise (PE) has in recent years been advocated as means to augment the effects of CBT for anxiety disorders. PE appears to reduce anxiety through other mechanisms than CBT, some of which might also have the potential to augment the effects of psychological treatment.

Aims: The current review aimed to summarize and discuss the current research status on CBT augmented with PE for anxiety.

Method: A systematic literature search was conducted in the databases PsychInfo, Medline and Web of Science to evaluate the potential augmentative effect of combining PE with CBT for anxiety disorders. These effects were intended to be evaluated in a meta-analysis, but findings from the few and diverse studies were better summarized in a systematic review.

Results: Eight articles were included in this review, of which two had no control group, while six had from two to four experimental arms. Six of the studies concluded in favour of benefits of add-on PE, while two studies found no added benefits of the combined interventions.

Conclusions: The combination of PE and CBT appears feasible. Add-on PE seems to be more beneficial for clinical populations, when administered regularly several times per week, across several weeks. Future studies should investigate further how and for whom to best combine PE and CBT.
\end{abstract}

Keywords: anxiety; CBT; cognitive behavioural therapy; physical exercise

\section{Introduction}

\section{Anxiety disorders}

Anxiety disorders are the most frequent of all mental disorders, with an estimated global current prevalence of 7.3\% (Baxter et al., 2013), and 12-month prevalence estimates ranging from 14\% (Wittchen et al., 2011) to $22.2 \%$ (Kessler et al., 2012), indicating that anxiety disorders affect a large proportion of the population across the lifetime, and are a large societal burden. If left untreated, most anxiety disorders have a chronic course (Craske et al., 2017), and often lead to a high degree of impairment for the individual and a great economic burden for society (Bandelow and Michaelis, 2015).

(C) The Author(s), 2021. Published by Cambridge University Press on behalf of the British Association for Behavioural and Cognitive Psychotherapies. This is an Open Access article, distributed under the terms of the Creative Commons Attribution licence (http://creativecommons.org/licenses/by/4.0/), which permits unrestricted re-use, distribution, and reproduction in any medium, provided the original work is properly cited. 
Cognitive behavioural therapy (CBT) and pharmacological treatments are the treatments of choice for anxiety disorders (National Institute for Health and Clinical Excellence, 2011, 2013), with both yielding significant symptom reduction for all anxiety disorders compared with control conditions (Bandelow et al., 2015; Mayo-Wilson et al., 2014). In this review, we will focus on the psychological treatment for anxiety disorders with the highest level of evidence, namely CBT (Bandelow et al., 2017; Kaczkurkin and Foa, 2015; Mayo-Wilson et al., 2014).

The effects of CBT treatment on anxiety disorders are widely studied and documented in several meta-analyses. CBT for anxiety disorders yield large effect sizes (Bandelow et al., 2015), ranging from small to large on CBT for different anxiety diagnoses compared with placebo for the different disorders (Carpenter et al., 2018; Wolitzky-Taylor et al., 2008), and with remission rates of $48 \%$ at posttreatment and $54 \%$ at follow-up assessments for intent to treat samples (Springer et al., 2018). Although the effectiveness of CBT for anxiety disorders is well documented, the reported findings indicate that half of patients do not achieve full remission, meaning that half of patients still qualify for an anxiety disorder or have anxiety symptoms above clinical cut-off at the end of treatment. In a review of non-adherence and non-response in the treatment of anxiety disorders, Taylor et al. (2012) report drop-out rates ranging from 9 to $21 \%$, and concluded that many patients fail to achieve clinically significant improvements. They suggest augmenting CBT with other interventions as an option for overcoming non-response (Taylor et al., 2012).

During the last decade, there has been increasing interest in how to optimize the efficacy of CBT. Studies have investigated add-on pharmacological interventions, such as using NMDA-partial agonist D-cycloserine to increase learning (Mataix-Cols et al., 2017; Pittig et al., 2016), and cognitive interventions such as executive skills training for late-life generalized anxiety disorder (Mohlman, 2008; Mohlman et al., 2003). The current review focuses on studies investigating the potential effects of physical exercise (PE) as an add-on intervention to CBT for anxiety disorders.

\section{Physical exercise}

PE has been thoroughly documented to be an efficacious treatment for depression (Kvam et al., 2016; Schuch et al., 2016), and in a recent meta-review, PE was found to be overall beneficial for a number of mental health problems (Czosnek et al., 2019). PE consists of both aerobic and resistance training, and can be performed in a variety of modalities such as running, walking and strength exercise training (American College of Sports Medicine, 2000). Rosenbaum et al. (2015) argue that PE is still widely under-utilized for mental health problems, while crosssectional studies indicate an association between low physical activity and increased anxiety levels (Goodwin, 2003; Stubbs et al., 2017). Several reviews and meta-analyses have summarized the effects of PE in treatment of anxiety disorders (see e.g. Asmundson et al., 2013; Aylett et al., 2018; Bartley et al., 2013; Kandola et al., 2018; Stonerock et al., 2015). These articles highlight different aspects of PE, and generally point to an anxiolytic effect of PE. In their review from 2013, Asmundson and colleagues identified that a wide variety of exercise modalities (walking, running, resistance training, yoga and tai chi) were related to anxiety reduction. Kandola and colleagues (2018) summarize that PE seems to be a beneficial and cost-effective intervention with anxiolytic effects, in addition to the beneficial physiological effects of PE.

The effect of exercise on anxiety reduction seems to be dependent on several factors. Previous literature suggests that exercise is best administered after exposure sessions (Roquet and Monfils, 2018; Tanner et al., 2018), that exercise needs to exceed 10 weeks in order for anxiety reductions to occur (Petruzzello et al., 1991), with a duration of at least 30 minutes (Herring et al., 2010), and that the optimal exercise frequency for anxiety reduction is three or four times per week (Wipfli et al., 2008). A recent meta-analysis also suggests that high intensity PE is more effective than low intensity for anxiety reduction (Aylett et al., 2018).

While it has been demonstrated that PE can effectively reduce levels of anxiety, it has been recommended as an add-on intervention to evidence-based treatments rather than a stand- 
alone treatment for anxiety disorders (Bartley et al., 2013; Stonerock et al., 2015). Several of the proposed mechanisms related to anxiety reduction from PE appear to differ from the mechanisms behind the effects of CBT and pharmacotherapies. Asmundson and colleagues (2013) and Kandola and colleagues (2018) summarize the proposed mechanisms for anxiety reduction for exercise. The physiological mechanisms include regulation of stress through the hypothalamicpituitary-adrenal axis, improving hippocampal plasticity and function, reducing the levels of inflammatory proteins, and regulation of neurotransmitters and neuropeptides such as brain derived neurotrophic factor (BDNF). The psychological mechanisms suggested are improved cognitive functioning, self-efficacy and mastery, improved sleep, improvements in positive affect and reduction in negative affect, exposure to bodily symptoms, reduced anxiety sensitivity and habituation. BDNF reliably increases from PE (Szuhany et al., 2015), and has specifically been highlighted as a potential mechanism for improving the effects of other exposure-based therapies, such as CBT (Kobayashi et al., 2005). The positive effects of PE on cognition and executive functions have also been related to the potential augmentative effects PE on CBT (Stavestrand et al., 2019), as effective executive functioning appears an important pre-requisite for effective cognitive restructuring (Mohlman, 2008; Yochim et al., 2013). As such, PE could potentially be a potent and beneficial add-on intervention to augment the effect of CBT or pharmacotherapies.

Few previous studies have investigated the combined effects of CBT and PE for anxiety and related disorders. A meta-analysis by Bernard and colleagues (2018) investigated the combined effects of CBT and PE for adults with chronic disease and concluded that their findings did not support an additive effect of $\mathrm{CBT}$ and $\mathrm{PE}$ for anxiety, depression, fatigue or pain. However, anxiety was not the primary target in this sample.

This review aims to summarize and discuss the current research status on CBT augmented with PE for anxiety. Although several reviews and meta-analyses have touched upon the issues we wanted to investigate, it seems to us that the specific strategy of combining PE with CBT for anxiety has not been the main focus in any of these articles. As such, we wanted to review existing literature to answer two separate research questions:

(1) By assessing both controlled and uncontrolled studies, is PE feasible as an add-on intervention to CBT for patients with anxiety in terms of treatment adherence and dropout rates?

(2) In patients suffering from anxiety, is adding PE to CBT more efficacious in reducing anxiety than either CBT alone or CBT combined with a control condition?

\section{Method}

We have applied the Population, Intervention, Comparison and Outcome Study (PICOS) (Tacconelli, 2010) framework to form the research questions and inform the literature search and selection of studies for this review.

\section{Identification of studies}

We conducted a systematic search using the databases PsycINFO, Medline and Web of Science. An overview of all the search terms, subject headings and mesh terms can be found in Appendix A (see Supplementary material). The search consisted of two search stems: 'anxiety disorders' and 'physical exercise'. All alternative search terms within the two search stems were combined with the Boolean operator 'OR'. We then combined the two search stems using the Boolean operator 'AND'. The final search was done on 10 August 2018 in the listed databases. We were also informed of subsequent publications based on the search strategy through email alerts from 
each of the databases. Email alerts were disabled on 19 November 2018. Where necessary information regarding study design or results was not provided in the article, the first author was contacted by email. The systematic search identified 13 reviews of PE and anxiety (Asmundson et al., 2013; Cavarretta et al., 2018; da Silva et al., 2009; DeBoer et al., 2012; de Souza Moura et al., 2015; Hales and Travis, 1987; Herring et al., 2010; Jayakody et al., 2014; Kandola et al., 2018; Kerr and Vlaswinkel, 1990; Martinsen, 2003; Powers et al., 2015; Stonerock et al., 2015). These reviews were assessed to identify additional relevant papers. Two articles (Lambert et al., 2007; Oldridge et al., 1991) were identified as potentially relevant, and these were evaluated according to the same criteria as articles identified through our own systematic search.

\section{Selection of studies}

Following duplicate removal, the first and third authors independently reviewed the titles and abstracts from the initial search and excluded the studies that did not fulfil the inclusion criteria. Any disagreements regarding inclusion were discussed and consensus was reached. Both authors then read the full text of the included studies. Studies assessed to meet inclusion criteria after reading the full text were included for the review. In cases of doubt, the second author was consulted for a third opinion regarding if the study met inclusion criteria.

\section{Inclusion criteria}

Only peer-reviewed articles published in English, Norwegian, Danish or Swedish language were included, due to the nationality of the authors. Studies were included in the review if they met the following criteria according to the PICOS framework (Tacconelli, 2010).

(1) Population: adults with anxiety disorders (specific phobia, panic disorder, social anxiety disorder, agoraphobia, generalized anxiety disorder) determined by either clinician assessment or questionnaire, or sub-clinical anxiety symptoms, i.e. elevated levels of anxiety below the clinical cut-off scores on the respective self-report measures.

(2) Intervention: PE as add-on intervention to CBT. PE interventions were operationalized according to the definition of $\mathrm{PE}$ as physical activity consisting of planned, structured and repetitive movements, aimed at improving and/or maintaining physical fitness (Caspersen et al., 1985). The specific content of PE included, but was not limited to, running, sports, resistance training and walking programmes. Interventions could be supervised or home-based. We made no restrictions regarding format, frequency, intensity or duration of the intervention.

(3) Comparison: the $\mathrm{PE}+\mathrm{CBT}$ intervention group was compared with $\mathrm{CBT}+$ control intervention, CBT alone, or no comparison.

(4) Outcome: change in anxiety levels as measured by change in score on self-report questionnaires or as determined through clinical interviews.

(5) Study: randomized and non-randomized clinical studies with multiple participants.

\section{Exclusion criteria}

Studies were excluded if anxiety was not the primary target for the intervention. Studies with several confounding interventions were excluded. Studies that described the PE intervention as mainly relaxation exercises (such as deep breathing exercises, progressive relaxation, meditation, mindfulness or low impact yoga) were excluded. Single case studies were excluded. 


\section{Quality assessment}

We assessed study quality using the Jadad scale for reporting randomized controlled trials (Jadad et al., 1996). The Jadad scale is intended to measure the quality of randomized controlled trials by focusing on random assignment, double blinding and the flow of patients. A thorough description of the Jadad scale, and assessment of each included study, can be found in Appendix B (see Supplementary material).

\section{Results}

\section{Characteristics of included studies}

We included eight studies with a total of 431 subjects for the planned meta-analysis (Bischoff et al., 2018; Cromarty et al., 2004; Gaudlitz et al., 2015; Hovland et al., 2015; Jacquart et al., 2017; McEntee and Halgin, 1999; Merom et al., 2008; Schwartz and Kaloupek, 1987). Figure 1 displays the selection of studies from a total of 11,437 articles identified through systematic search.

Table 1 displays characteristics of the included studies. The studies varied greatly regarding study design, sample size, diagnoses, clinical $v s$ non-clinical population, pharmacological treatment, duration of intervention, type of CBT intervention, type of PE intervention, administration of PE intervention and study quality. The studies were considered too few and diverse to be meaningfully assessed in a meta-analysis and were instead evaluated in a systematic review.

\section{Design}

Two studies were pilot studies without a control group (Cromarty et al., 2004; Hovland et al., 2015). One 'pilot RCT' had two experimental arms comparing add-on PE to add-on educational group (Merom et al., 2008). Three RCTs had two experimental arms comparing either add-on PE with add-on sedentary intervention (Jacquart et al., 2017), or add-on moderate intensity PE to add-on low intensity PE (Bischoff et al., 2018; Gaudlitz et al., 2015). Two RCTs had four experimental arms, comparing CBT+PE to CBT alone, PE alone, or placebo (McEntee and Halgin, 1999; Schwartz and Kaloupek, 1987).

\section{Sample}

Three studies had mainly non-clinical samples recruited from universities and surrounding communities (Jacquart et al., 2017; McEntee and Halgin, 1999; Schwartz and Kaloupek, 1987). Five studies had clinical samples recruited from specialized outpatient clinics (Bischoff et al., 2018; Cromarty et al., 2004; Gaudlitz et al., 2015; Hovland et al., 2015; Merom et al., 2008). The specific diagnoses are listed in Table 1.

\section{Psychological interventions}

The included studies varied regarding the psychological interventions employed. Four studies used a group-based CBT format (Cromarty et al., 2004; Gaudlitz et al., 2015; McEntee and Halgin, 1999; Merom et al., 2008), and four studies used individual CBT (Bischoff et al., 2018; Hovland et al., 2015; Jacquart et al., 2017; Schwartz and Kaloupek, 1987). The individually administered CBT interventions included exposure-based CBT (Bischoff et al., 2018), internetbased CBT (Hovland et al., 2015), virtual reality exposure therapy (Jacquart et al., 2017) and imaginal exposure therapy (Schwartz and Kaloupek, 1987). 


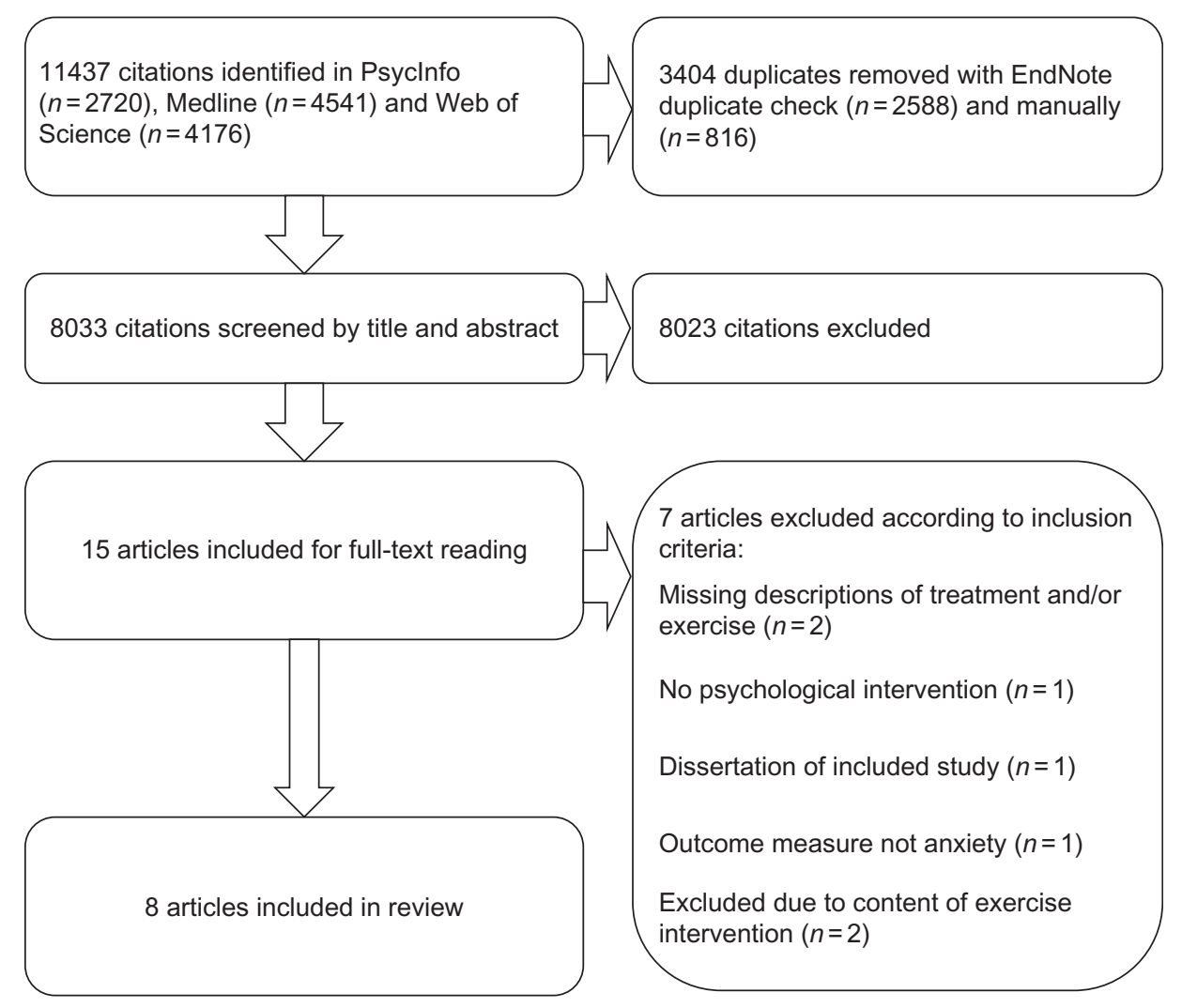

Figure 1. Flow chart of selection process.

\section{PE interventions}

The included studies varied in terms of both the content and administration of the exercise interventions.

Content. Three studies described the intervention as moderate intensity aerobic exercise (Bischoff et al., 2018; Gaudlitz et al., 2015; McEntee and Halgin, 1999), one as an hour in the gym with experiments to test catastrophic beliefs (Cromarty et al., 2004), one as endurance and interval training with high intensity (Hovland et al., 2015), one as walking (Merom et al., 2008), one as vigorous aerobic exercise (Jacquart et al., 2017), and one study describes the exercise intervention as ergometer cycling with gradually increasing workload (Schwartz and Kaloupek, 1987). Four studies (Bischoff et al., 2018; Gaudlitz et al., 2015; Hovland et al., 2015; McEntee and Halgin, 1999) based their training programmes on recommendations by the American College of Sports Medicine (ACSM) (2000), and one study (Merom et al., 2008) based the training programme on the Australian physical activity recommendations (Commonwealth Department of Health and Ageing, 1999).

Administration. Four studies administered PE as an add-on intervention in parallel to the CBT intervention, during the full treatment period (Gaudlitz et al., 2015; Hovland et al., 2015; McEntee and Halgin, 1999; Merom et al., 2008). In these studies, the participants exercised between three and five times per week, and the add-on PE can as such be labelled as 'regular PE'. One of these studies also specifically instructed participants to keep exercising one month after end of treatment (Gaudlitz et al., 2015). 
Table 1. Overview of included studies, characteristics and conclusions

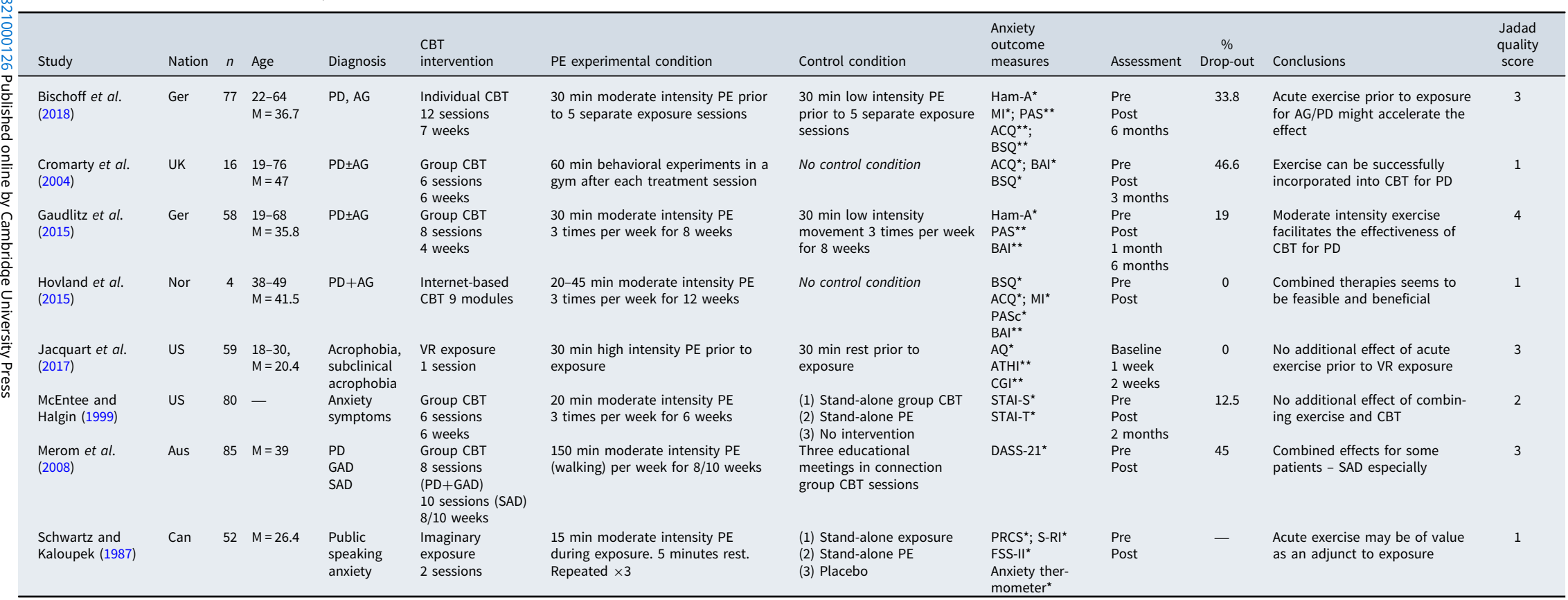

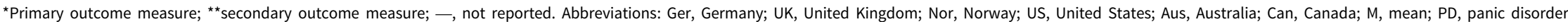

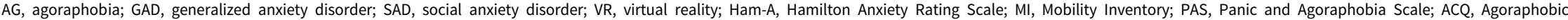

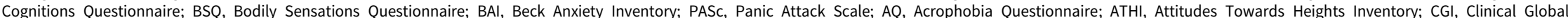

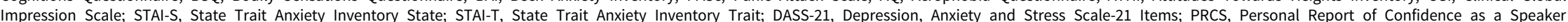
questionnaire; S-RI, S-R Inventory of Anxiousness; FSS-II, Fear Survey Schedule II. 
Three studies administered PE in connection with exposure sessions. In two studies, participants exercised prior to the exposure session (Bischoff et al., 2018; Jacquart et al., 2017), and in the third of these studies participants exercised during the exposure session (Schwartz and Kaloupek, 1987). The add-on PE in these studies targeted enhancing the effects of exposure therapy by using the beneficial effects of acute PE and can therefore be labelled 'acute PE'.

In one study, patients with panic disorder went to the gym directly after CBT sessions, to test catastrophic beliefs regarding their bodily anxiety symptoms (Cromarty et al., 2004). Thus, this use of PE can be labelled 'behavioural experiment'.

\section{Control conditions}

Two of the included studies were pilot or feasibility studies without a control condition (Cromarty et al., 2004; Hovland et al., 2015).

Six of the included studies had control conditions. As with the PE interventions, the control conditions vary regarding content and administration.

Content. Two studies (Bischoff et al., 2018; Gaudlitz et al., 2015) had low-intensity PE as control condition. One study had educational group meetings focused on healthy eating (Merom et al., 2008). One study had a weekly discussion group unrelated to the treatment (McEntee and Halgin, 1999). In one study, the control group sat still on the ergometer bike, while listening to the neutral imagery (Schwartz and Kaloupek, 1987). In the study by Jacquart and colleagues (2017), participants in the control condition watched a comedy television show of their choosing, prior to treatment.

Administration. Four studies administered the control condition as an add-on to the therapeutic intervention (Bischoff et al., 2018; Jacquart et al., 2017; Merom et al., 2008). Two studies administered the control condition without any therapeutic intervention (McEntee and Halgin, 1999; Schwartz and Kaloupek, 1987). All the studies with a control condition administered it with the same frequency as the supervised PE intervention, with the exception of Merom and colleagues (2008), who had three educational meetings as control for weekly PE. The unsupervised PE was not controlled for in any of the studies.

\section{Feasibility and drop-out}

Among the included studies, four studies (Gaudlitz et al., 2015; Hovland et al., 2015; Jacquart et al., 2017; McEntee and Halgin, 1999) reported overall drop-out rates within or below the expected drop-out range for anxiety disorders of 9-21\% identified in the review by Taylor and colleagues (2012). One study did not mention drop-out rates (Schwartz and Kaloupek, 1987), while three studies had high post-measure drop-out rates of 33.8\% (Bischoff et al., 2018), 46.6\% (Cromarty et al., 2004) and 45\% (Merom et al., 2008). Six of the eight studies (Bischoff et al., 2018; Gaudlitz et al., 2015; Hovland et al., 2015; Jacquart et al., 2017; McEntee and Halgin, 1999; Schwartz and Kaloupek, 1987) indicated that the combination was feasible for the patients and that the combination did not affect treatment compliance.

\section{Efficacy of PE as an add-on intervention to CBT}

Studies without a control group

Hovland and colleagues (2015) have published preliminary results of a feasibility study on combining internet-based CBT with PE for panic disorder. Cromarty and colleagues (2004) conducted a pilot study on group CBT combined with PE for participants with panic disorder with or without agoraphobia. Both studies concluded that the combination of PE and CBT is 
meaningful and potentially beneficial. However, they are careful not to draw firm conclusions due to the nature of the study designs.

\section{Studies with a control group}

Two studies clearly concluded with no beneficial add-on effects of PE to CBT for anxiety (Jacquart et al., 2017; McEntee and Halgin, 1999). Two studies had ambiguous results and argued that PE potentially has beneficial add-on effects (Bischoff et al., 2018; Schwartz and Kaloupek, 1987). Bischoff and colleagues (2018) identified a trend towards significance on general anxiety measures in favour of the addition of moderate intensity compared with the addition of low intensity exercise. The difference faded at follow-up. Schwartz and Kaloupek (1987) found additional effects of exercise on imaginal exposure limited to subjective units of distress (Wolpe, 1969). Two studies clearly conclude with beneficial add-on effects of PE to CBT for anxiety disorders (Gaudlitz et al., 2015; Merom et al., 2008), with add-on effects on general, but not disorder-specific, anxiety self-report measures.

\section{Discussion}

This systematic review is the first study to summarize the feasibility and efficacy of combining PE with CBT for participants primarily suffering from anxiety or elevated levels of anxiety. Overall, the reviewed studies suggest that the combination of PE and CBT is feasible for patients, and that $\mathrm{PE}$ might be beneficial as an add-on intervention to CBT. However, several factors might influence whether PE has an augmentative effect on CBT, and the following sections discuss the feasibility of this combination, and under which circumstances PE might yield a beneficial add-on effect.

The included studies in the present review suggest that the combination of PE and CBT is feasible, and generally with acceptable adherence and attrition rates. None of the studies reported adverse effects of $\mathrm{PE}$. The studies with drop-out rates above the expected rate of 9-21\% (Taylor et al., 2012) were all studies on clinical populations (Bischoff et al., 2018; Cromarty et al., 2004; Merom et al., 2008), which could question the feasibility of this combination for clinical populations. Although the study by Bischoff and colleagues (2018) reported high drop-out, only two participants dropped out due to PE-related reasons. However, both Cromarty and colleagues (2004) and Merom and colleagues (2008) suggested that the relatively high drop-out rates reported in their studies might be related to the addition of PE in combination with CBT. Merom and colleagues (2008) suggested that people who were more active at baseline were more likely to complete treatment. The two other studies with clinical populations (Gaudlitz et al., 2015; Hovland et al., 2015) reported acceptable drop-out rates. Gaudlitz and colleagues (2015) reported decreasing response rates to follow-up measures in the follow-up assessments compared with post-treatment assessment, but the adherence to follow-up was better for the CBT+PE group than for the control group. This might indicate that drop-out was related to other factors than PE. The large variation in drop-out rates, and heterogeneity of studies, makes it difficult to draw firm conclusions regarding the feasibility of $\mathrm{PE}$ as an add-on intervention to CBT for patients with anxiety. Future studies should continue to report and investigate drop-out and adverse effects, to further establish the feasibility of the combined interventions.

The efficacy of adding PE interventions to CBT seem to depend on a number of potential moderating variables. We will discuss potential factors related to the design of the $\mathrm{PE}$ interventions, namely the administration of add-on PE, the duration of $\mathrm{PE}$ sessions and $\mathrm{PE}$ intensity, and factors related to the design of the included studies, such as sample, diagnoses, follow-up data and study quality. The few and diverse studies included in this review makes it hard to draw conclusions regarding a number of potentially relevant factors, including age, gender, format (individual or group-based PE), anxiety outcome measures and culture. 
Regarding the administration of add-on PE, four studies administered the PE intervention several times per week, across six or more weeks (Gaudlitz et al., 2015; Hovland et al., 2015; McEntee and Halgin, 1999; Merom et al., 2008). Of these four studies, only one (McEntee and Halgin, 1999) concluded with no beneficial effects of PE. This might indicate that PE administered regularly across several weeks could be a beneficial add-on intervention. The studies also indicated that longer duration of PE interventions yielded more of an additive effect of PE (Gaudlitz et al., 2015; Merom et al., 2008). This is in line with previous literature suggesting that PE frequency of three or four times per week (Wipfli et al., 2008) and across at least 10 weeks (Petruzzello et al., 1991) is beneficial for anxiety reduction.

Of the three studies investigating the effects of acutely administered PE in connection to exposure sessions, one found a trend towards significance favouring add-on PE (Bischoff et al., 2018), while the two others found no add-on effects (Jacquart et al., 2017; Schwartz and Kaloupek, 1987). The lack of efficacy could be explained by administering the PE intervention before or during exposure, rather than after exposure, as PE after exposure has been identified as beneficial for memory consolidation (Roquet and Monfils, 2018; Tanner et al., 2018). However, other factors related to study design can also explain these results.

The efficacy of $\mathrm{PE}$ as behavioural experiments remains unclear, as the only study administrating PE as behavioural experiments (Cromarty et al., 2004) had high drop-out, poor study design, no control group, and found lower effect sizes than estimated effect sizes with CBT for panic disorder in general (Bandelow et al., 2015).

Future studies should investigate whether PE administered regularly over time has more benefits for anxiety reduction than other ways of administrating PE, such as PE in relation to exposure sessions or $\mathrm{PE}$ as behavioural experiments. Furthermore, studies investigating the administration of PE in relation to exposure therapy could test the hypothesis that add-on PE after exposure sessions could enhance treatment effects.

All studies except McEntee and Halgin (1999) administered bouts of PE with a duration of at least 30 minutes, which corresponds to both ACSM recommendations (2000) and the findings of Herring and colleagues (2010). As such, all the studies that found beneficial effects of add-on PE adhered to ACSM recommendations. However, differences in PE session duration do not seem to explain differences in add-on PE efficacy between the included studies in this review.

Regarding the role of intensity, only two studies compared two specified intensities of PE. Gaudlitz and colleagues (2015) found the addition of moderate PE to be superior to the addition of low intensity PE, while Bischoff and colleagues (2018) found no difference. Aylett and colleagues (2018) have identified PE intensity as a predictor for anxiety reduction when $\mathrm{PE}$ is administered as a stand-alone treatment. As such, future studies should investigate if PE intensity is a moderating variable in anxiety reduction also when PE is administered as an add-on intervention.

Due to the few available RCTs, we included studies with anxiety disorders according to DSM-V (American Psychiatric Association, 2013), as well as studies with CBT treatment for non-clinical anxiety. In the reviewed studies, three studies had non-clinical or sub-clinical samples (Jacquart et al., 2017; McEntee and Halgin, 1999; Schwartz and Kaloupek, 1987). All these studies indicated no or limited effects of PE as an add-on intervention to CBT. The lack of findings for non-clinical samples could be explained by lower levels of anxiety symptoms and thereby difficulties in detecting effects of add-on interventions. There could also be a ceiling effect, in which participants with sub-clinical symptoms already respond well to the main intervention.

A ceiling effect could also explain some of the differences in the studies with clinical samples. Remission rates and effect sizes of CBT are largest for generalized anxiety disorder, and smallest for social anxiety (Carpenter et al., 2018; Springer et al., 2018). Thus, it is noteworthy that the patients with the greatest anxiety reduction in the study by Merom and colleagues (2008), were patients with generalized anxiety disorder, who were also the group that exercised the least. Furthermore, the patients with social anxiety disorder appeared to benefit the most from 
the added PE in this study. The apparent lack of add-on effect of PE for the patients with clinical acrophobia in the study by Jacquart and colleagues (2017), could be explained by a ceiling effect for an already effective treatment of specific phobias (Wolitzky-Taylor et al., 2008). As such, we suggest that future research on add-on interventions, such as $\mathrm{PE}$, focuses on target populations and disorders where there is significant room for improvement of treatment efficacy.

For the RCT studies consisting of clinical samples, panic disorder with or without agoraphobia has been most studied, and the most promising add-on effects have been found for this disorder (Bischoff et al., 2018; Gaudlitz et al., 2015; Merom et al., 2008). For panic disorder, the standard treatment already involves interoceptive exposure to bodily symptoms (Pompoli et al., 2016), much like the symptoms experienced through exercise. The addition of exercise could act as an extension of this exposure. These findings might suggest that PE could be a beneficial addon intervention to CBT for patients with panic disorder, but the small number of studies needs to be taken into consideration. More studies with different anxiety disorders are needed in order to draw conclusions regarding the role of different anxiety disorders and potential ceiling effects.

Only two of the reviewed studies presented 6-month follow-up data (Bischoff et al., 2018; Gaudlitz et al., 2015). In the study by Gaudlitz and colleagues (2015), the differences in symptom reductions between moderate intensity $\mathrm{PE}$ and movements with little strain did not become apparent immediately after the treatment, and only became significant at 6-month follow-up. Bischoff and colleagues (2018) found no differences at 6-month follow-up. This raises the issue that the added beneficial effects of $\mathrm{PE}$, or lack of thereof, might become evident only over time. Future studies should include long-term follow-up assessments.

\section{Limitations}

We conducted a literature search with a broad search strategy, in order to find as many relevant articles as possible, but we are aware that we might not have included all relevant studies. The authors' language proficiencies limit available articles, and could thus have influenced which articles that were included for the review. We are aware of one Brazilian article regarding CBT combined with PE that was excluded due to language (Sardinha et al., 2011). Another limitation to methodology is the lack of measuring inter-rater reliability in the study selection, as we evaluated the search results manually without review software.

This review is based on eight studies, with heterogeneous design and methodologies. The included studies are characterized by small samples, variability in the presence and quality of control groups, lack of information concerning randomization and potential confounding variables such as psychotropic medication, lack of descriptions of potential underlying mechanisms of change, as well as mixed samples, making it harder to draw conclusions on a diagnostic level. Only one study had a Jadad score of 4; the remaining scored 3 or below, meaning that the general quality of the studies were weak (see Appendix $B$ in the Supplementary material). Due to the limited number of available studies, we did not exclude studies based on quality. The results should be interpreted with this in mind. Although we intended to do a meta-analysis, we found the data material insufficient for this approach. As such, we cannot draw any quantitative conclusions regarding the augmentative effects of PE, or adequately assess the impact of the factors discussed here.

We have included studies with measures of anxiety as outcome measures. We have not included studies that investigate biological mechanisms and changes related to PE, although hypotheses regarding beneficial effects of PE often have biological underpinnings. As such, we cannot draw conclusions regarding the relevance of such variables. Future studies should assess biological variables that could play a role in the augmentative effect of PE, in addition to investigating the psychological mechanisms of change. 


\section{Conclusions and further directions}

The reviewed literature indicates that the combination of PE and CBT treatment is feasible, and the addition of PE does not appear to affect treatment adherence. The effect of PE may be dependent on several factors. In the reviewed studies, PE seems to be a beneficial add-on strategy for clinical populations, rather than non-clinical samples. Regular PE, in which PE is administered several times per week, across several weeks, appears to be the most promising strategy. The next steps in research are high quality studies to determine under which circumstances PE can be applied as a beneficial add-on intervention to CBT; specifically, whether different administration strategies for combining PE and CBT yield different results, the effect of PE intensity, and the efficacy of add-on PE for populations and diagnoses with poorer response to stand-alone CBT. Such steps in research, in combination with research on when to apply $\mathrm{PE}$ as a stand-alone intervention, can ensure effective and strategic use of PE in mental health care.

Acknowledgements. Thanks to staff at the University Library of Bergen for assistance in developing the search strategy.

Financial support. The publishing of the article was funded by Diakonhjemmet Hospital. The funder had no role in the conduct or results of the study.

Conflicts of interest. None.

Data availability. The data that support the findings of this study are available from the corresponding author (K.P.F.) upon reasonable request.

Supplementary material. To view supplementary material for this article, please visit https://doi.org/10.1017/ S1352465821000126

\section{References}

American College of Sports Medicine (2000). ACSM 's Guidelines for Exercise Testing and Prescription. Philadelphia, USA: Lippincott Williams \& Wilkins.

American Psychiatric Association (2013). Diagnostic and Statistical Manual of Mental Disorders (5th edn; DSM-V). Washington, DC, USA.

Asmundson, G. J., Fetzner, M. G., DeBoer, L. B., Powers, M. B., Otto, M. W., \& Smits, J. A. (2013). Let's get physical: a contemporary review of the anxiolytic effects of exercise for anxiety and its disorders. Depression and Anxiety, 30, 362-373. https://doi.org/10.1002/da.22043

Aylett, E., Small, N., \& Bower, P. (2018). Exercise in the treatment of clinical anxiety in general practice - a systematic review and meta-analysis. BMC Health Services Research, 18, 559. https://doi.org/10.1186/s12913-018-3313-5

Bandelow, B., \& Michaelis, S. (2015). Epidemiology of anxiety disorders in the 21st century. Dialogues in Clinical Neuroscience, 17, 327-335

Bandelow, B., Michaelis, S., \& Wedekind, D. (2017). Treatment of anxiety disorders. Dialogues in Clinical Neuroscience, 19, 93-107

Bandelow, B., Reitt, M., Röver, C., Michaelis, S., Görlich, Y., \& Wedekind, D. (2015). Efficacy of treatments for anxiety disorders: a meta-analysis. International Clinical Psychopharmacology, 30, 183-192. https://doi.org/10.1097/yic. 0000000000000078

Bartley, C. A., Hay, M., \& Bloch, M. H. (2013). Meta-analysis: aerobic exercise for the treatment of anxiety disorders. Progress in Neuro-Psychopharmacology \& Biological Psychiatry, 45, 34-39. https://doi.org/10.1016/j.pnpbp.2013.04.016

Baxter, A. J., Scott, K. M., Vos, T., \& Whiteford, H. A. (2013). Global prevalence of anxiety disorders: a systematic review and meta-regression. Psychological Medicine, 43, 897-910. https://doi.org/10.1017/S003329171200147X

Bernard, P., Romain, A. J., Caudroit, J., Chevance, G., Carayol, M., Gourlan, M., . . \& Moullec, G. (2018). Cognitive behavior therapy combined with exercise for adults with chronic diseases: systematic review and meta-analysis. Health Psychology, 37, 433-450. https://doi.org/10.1037/hea0000578

Bischoff, S., Wieder, G., Einsle, F., Petzold, M. B., Jansen, C., Mumm, J. L., . . \& Strohle, A. (2018). Running for extinction? Aerobic exercise as an augmentation of exposure therapy in panic disorder with agoraphobia. Journal of Psychiatric Research, 101, 34-41. https://doi.org/10.1016/j.jpsychires.2018.03.001

Carpenter, J., Andrews, L.A., Witcraft, S., Powers, M., Smits, J., \& Hofmann, S. (2018). Cognitive behavioral therapy for anxiety and related disorders: a meta-analysis of randomized placebo-controlled trials. Depression and Anxiety, 35. https:// doi.org/10.1002/da. 22728 
Caspersen, C. J., Powell, K. E., \& Christenson, G. M. (1985). Physical activity, exercise, and physical fitness: definitions and distinctions for health-related research. Public Health Reports, 100, 126-131.

Cavarretta, D. J., Hall, E. E., \& Bixby, W. R. (2018). The acute effects of resistance exercise on affect, anxiety, and mood practical implications for designing resistance training programs. International Review of Sport and Exercise Psychology. https://doi.org/10.1080/1750984X.2018.1474941

Commonwealth Department of Health and Ageing (1999). National physical activity guidelines. Canberra, Australia: Commonwealth Department of Health and Ageing.

Craske, M. G., Stein, M. B., Eley, T. C., Milad, M. R., Holmes, A., Rapee, R. M., \& Wittchen, H.-U. (2017). Anxiety disorders. Nature Reviews Disease Primers, 3, 17024. https://doi.org/10.1038/nrdp.2017.24

Cromarty, P., Robinson, G., Callcott, P., \& Freeston, M. (2004). Cognitive therapy and exercise for panic and agoraphobia in primary care: pilot study and service development. Behavioural and Cognitive Psychotherapy, 32, 371-374. https://doi.org/ 10.1017/S1352465804001456

Czosnek, L., Lederman, O., Cormie, P., Zopf, E., Stubbs, B., \& Rosenbaum, S. (2019). Health benefits, safety and cost of physical activity interventions for mental health conditions: a meta-review to inform translation efforts. Mental Health and Physical Activity, 16, 140-151. https://doi.org/10.1016/j.mhpa.2018.11.001

da Silva, T. L., Ravindran, L. N., \& Ravindran, A. V. (2009). Yoga in the treatment of mood and anxiety disorders: a review. Asian Journal of Psychiatry, 2, 6-16. https://doi.org/10.1016/j.ajp.2008.12.002

de Souza Moura, A. M., Lamego, M. K., Paes, F., Ferreira Rocha, N. B., Simoes-Silva, V., Rocha, S. A., ... \& Machado, S. (2015). Effects of aerobic exercise on anxiety disorders: a systematic review. CNS \& Neurological Disorders Drug Targets, 14, 1184-1193. https://doi.org/10.2174/1871527315666151111121259

DeBoer, L. B., Powers, M. B., Utschig, A. C., Otto, M. W., \& Smits, J. A. (2012). Exploring exercise as an avenue for the treatment of anxiety disorders. Expert Review of Neurotherapeutics, 12, 1011-1022. https://doi.org/10.1586/ern.12.73

Gaudlitz, K., Plag, J., Dimeo, F., \& Ströhle, A. (2015). Aerobic exercise training facilitates the effectiveness of cognitive behavioral therapy in panic disorder. Depression and Anxiety, 32, 221-228. https://doi.org/10.1002/da.22337

Goodwin, R. D. (2003). Association between physical activity and mental disorders among adults in the United States. Preventive Medicine, 36, 698-703. https://doi.org/10.1016/S0091-7435(03)00042-2

Hales, R. E., \& Travis, T. W. (1987). Exercise as a treatment option for anxiety and depressive disorders. Military Medicine, 152, 299-302

Herring, M. P., O’Connor, P. J., \& Dishman, R. K. (2010). The effect of exercise training on anxiety symptoms among patients: a systematic review. Archives of Internal Medicine, 170, 321-331. https://doi.org/10.1001/archinternmed. 2009.530

Hovland, A., Johansen, H., Sjobo, T., Vollestad, J., Nordhus, I. H., Pallesen, S., .. \& Nordgreen, T. (2015). A feasibility study on combining internet-based cognitive behaviour therapy with physical exercise as treatment for panic disorder treatment protocol and preliminary results. Cognitive Behaviour Therapy, 44, 275-287. https://doi.org/10.1080/16506073. 2015.1022596

Jacquart, J., Roquet, R. F., Papini, S., Powers, M. B., Rosenfield, D., Smits, J. A. J., \& Monfils, M. H. (2017). Effects of acute exercise on fear extinction in rats and exposure therapy in humans: null findings from five experiments. Journal of Anxiety Disorders, 50, 76-86. https://doi.org/10.1016/j.janxdis.2017.05.010

Jadad, A. R., Moore, R. A., Carroll, D., Jenkinson, C., Reynolds, D. J. M., Gavaghan, D. J., \& McQuay, H. J. (1996). Assessing the quality of reports of randomized clinical trials: is blinding necessary? Controlled Clinical Trials, 17, 1-12. https://doi.org/10.1016/0197-2456(95)00134-4

Jayakody, K., Gunadasa, S., \& Hosker, C. (2014). Exercise for anxiety disorders: systematic review. British Journal of Sports Medicine, 48, 187-196. https://doi.org/10.1136/bjsports-2012-091287

Kaczkurkin, A. N., \& Foa, E. B. (2015). Cognitive-behavioral therapy for anxiety disorders: an update on the empirical evidence. Dialogues in Clinical Neuroscience, 17, 337-346

Kandola, A., Vancampfort, D., Herring, M., Rebar, A., Hallgren, M., Firth, J., \& Stubbs, B. (2018). Moving to beat anxiety: epidemiology and therapeutic issues with physical activity for anxiety. Current Psychiatry Reports, 20, 63. https://doi.org/10. 1007/s11920-018-0923-x

Kerr, J. H., \& Vlaswinkel, E. H. (1990). Effects of exercise on anxiety: a review. Anxiety Research, 2, 309-321. https://doi.org/ $10.1080 / 08917779008248736$

Kessler, R. C., Petukhova, M., Sampson, N. A., Zaslavsky, A. M., \& Wittchen, H.-U. (2012). Twelve-month and lifetime prevalence and lifetime morbid risk of anxiety and mood disorders in the United States. International Journal of Methods in Psychiatric Research, 21, 169-184. https://doi.org/10.1002/mpr.1359

Kobayashi, K., Shimizu, E., Hashimoto, K., Mitsumori, M., Koike, K., Okamura, N., . . \& Iyo, M. (2005). Serum brainderived neurotrophic factor (BDNF) levels in patients with panic disorder: as a biological predictor of response to group cognitive behavioral therapy. Progress in Neuro-Psychopharmacology and Biological Psychiatry, 29, 658-663. https://doi. org/10.1016/j.pnpbp.2005.04.010

Kvam, S., Kleppe, C. L., Nordhus, I. H., \& Hovland, A. (2016). Exercise as a treatment for depression: a meta-analysis. Journal of Affective Disorders, 202, 67-86. https://doi.org/10.1016/j.jad.2016.03.063 
Lambert, R. A., Harvey, I., \& Poland, F. (2007). A pragmatic, unblinded randomised controlled trial comparing an occupational therapy-led lifestyle approach and routine GP care for panic disorder treatment in primary care. Journal of Affective Disorders, 99, 63-71. https://doi.org/10.1016/j.jad.2006.08.026

Martinsen, E. W. (2003). Running away from anxiety and depression? Tidsskrift for Norsk Psykologforening, 40, 676-681

Mataix-Cols, D., Fernández de la Cruz, L., Monzani, B., Rosenfield, D., Andersson, E., Pérez-Vigil, A., ... \& Thuras, T. (2017). D-Cycloserine augmentation of exposure-based cognitive behavior therapy for anxiety, obsessive-compulsive, and posttraumatic stress disorders: a systematic review and meta-analysis of individual participant data. JAMA Psychiatry, 74, 501-510. https://doi.org/10.1001/jamapsychiatry.2016.3955

Mayo-Wilson, E., Dias, S., Mavranezouli, I., Kew, K., Clark, D. M., Ades, A. E., \& Pilling, S. (2014). Psychological and pharmacological interventions for social anxiety disorder in adults: a systematic review and network meta-analysis. The Lancet Psychiatry, 1, 368-376. https://doi.org/10.1016/S2215-0366(14)70329-3

McEntee, D. J., \& Halgin, R. P. (1999). Cognitive group therapy and aerobic exercise in the treatment of anxiety. Journal of College Student Psychotherapy, 13, 37-55. https://doi.org/10.1300/J035v13n03_04

Merom, D., Phongsavan, P., Wagner, R., Chey, T., Marnane, C., Steel, Z., . . \& Bauman, A. (2008). Promoting walking as an adjunct intervention to group cognitive behavioral therapy for anxiety disorders - a pilot group randomized trial. Journal of Anxiety Disorders, 22, 959-968. https://doi.org/10.1016/j.janxdis.2007.09.010

Mohlman, J. (2008). More power to the executive? A preliminary test of CBT plus executive skills training for treatment of late-life GAD. Cognitive and Behavioral Practice, 15, 306-316. https://doi.org/10.1016/j.cbpra.2007.07.002

Mohlman, J., Gorenstein, E. E., Kleber, M., de Jesus, M., Gorman, J. M., \& Papp, L. A. (2003). Standard and enhanced cognitive-behavior therapy for late-life generalized anxiety disorder: two pilot investigations. American Journal of Geriatric Psychiatry, 11, 24-32. https://doi.org/10.1097/00019442-200301000-00005

National Institute for Health and Clinical Excellence (2011). Generalised anxiety disorder and panic disorder (with or without agoraphobia) in adults. Clinical guideline 113. London, UK. Retrieved from: http://www.guidance.nice.org.uk/ CG113

National Institute for Health and Clinical Excellence (2013). Social anxiety disorder: recognition, assessment and treatment. Clinical guideline 159. London, UK. Retrieved from: http://www.guidance.nice.org.uk/CG159

Oldridge, N., Guyatt, G., Jones, N., Crowe, J., Singer, J., Feeny, D., . . \& Torrance, G. (1991). Effects on quality of life with comprehensive rehabilitation after acute myocardial infarction. American Journal of Cardiology, 67, 1084-1089. https://doi. org/10.1016/0002-9149(91)90870-Q

Petruzzello, S. J., Landers, D. M., Hatfield, B. D., Kubitz, K. A., \& Salazar, W. (1991). A meta-analysis on the anxietyreducing effects of acute and chronic exercise. Sports Medicine, 11, 143-182. https://doi.org/10.2165/00007256-19911103000002

Pittig, A., van den Berg, L., \& Vervliet, B. (2016). The key role of extinction learning in anxiety disorders: behavioral strategies to enhance exposure-based treatments. Current Opinion in Psychiatry, 29, 39-47. https://.doi.org/10.1097/yco. 000000000000220

Pompoli, A., Furukawa, T. A., Imai, H., Tajika, A., Efthimiou, O., \& Salanti, G. (2016). Psychological therapies for panic disorder with or without agoraphobia in adults: a network meta-analysis. Cochrane Database of Systematic Reviews, 4. https://doi.org/10.1002/14651858.CD011004.pub2

Powers, M. B., Asmundson, G. J., \& Smits, J. A. (2015). Exercise for mood and anxiety disorders: the state-of-the science. Cognitive Behaviour Therapy, 44, 237-239. https://doi.org/10.1080/16506073.2015.1047286

Roquet, R., \& Monfils, M.-H. (2018). Does exercise augment operant and Pavlovian extinction: a meta-analysis. Journal of Psychiatric Research, 96, 73-93. https://doi.org/10.1016/j.jpsychires.2017.09.018

Rosenbaum, S., Tiedemann, A., Ward, P. B., Curtis, J., \& Sherrington, C. (2015). Physical activity interventions: an essential component in recovery from mental illness. British Journal of Sports Medicine, 49, 1544-1545. https://doi.org/ 10.1136/bjsports-2014-094314

Sardinha, A., de Araujo, C. G. S., \& Nardi, A. E. (2011). Interval aerobic training as a tool in the cognitive-behavioral treatment of panic disorder. Jornal Brasileiro de Psiquiatria, 60, 227-230. https://doi.org/10.1590/S004720852011000300013

Schuch, F. B., Vancampfort, D., Richards, J., Rosenbaum, S., Ward, P. B., \& Stubbs, B. (2016). Exercise as a treatment for depression: a meta-analysis adjusting for publication bias. Journal of Psychiatric Research, 77, 42-51. https://doi.org/10. 1016/j.jpsychires.2016.02.023

Schwartz, S. G., \& Kaloupek, D. G. (1987). Acute exercise combined with imaginal exposure as a technique for anxiety reduction. Canadian Journal of Behavioural Science/Revue canadienne des sciences du comportement, 19, 151-166. https://doi.org/10.1037/h0080012

Springer, K. S., Levy, H. C., \& Tolin, D. F. (2018). Remission in CBT for adult anxiety disorders: a meta-analysis. Clinical Psychology Review, 61, 1-8. https://doi.org/10.1016/j.cpr.2018.03.002

Stavestrand, S. H., Sirevåg, K., Nordhus, I. H., Sjøbø, T., Endal, T. B., Nordahl, H. M., . . \& Hovland, A. (2019). Physical exercise augmented cognitive behaviour therapy for older adults with generalised anxiety disorder (PEXACOG): study protocol for a randomized controlled trial. Trials, 20, 174. https://doi.org/10.1186/s13063-019-3268-9 
Stonerock, G. L., Hoffman, B. M., Smith, P. J., \& Blumenthal, J. A. (2015). Exercise as treatment for anxiety: systematic review and analysis. Annals of Behavioral Medicine, 49, 542-556. https://doi.org/10.1007/s12160-014-9685-9

Stubbs, B., Koyanagi, A., Hallgren, M., Firth, J., Richards, J., Schuch, F., . . \& Vancampfort, D. (2017). Physical activity and anxiety: a perspective from the World Health Survey. Journal of Affective Disorders, 208, 545-552. https://doi.org/10. 1016/j.jad.2016.10.028

Szuhany, K.L., Bugatti, M., \& Otto, M.W. (2015). A meta-analytic review of the effects of exercise on brain-derived neurotrophic factor. Journal of Psychiatric Research, 60, 56-64. https://doi.org/10.1016/j.jpsychires.2014.10.003

Tacconelli, E., (2010). Systematic reviews: CRD's guidance for undertaking reviews in health care. The Lancet Infectious Diseases, 10, 226. https://doi.org/10.1016/S1473-3099(10)70065-7

Tanner, M. K., Hake, H. S., Bouchet, C. A., \& Greenwood, B. N. (2018). Running from fear: exercise modulation of fear extinction. Neurobiology of Learning and Memory, 151, 28-34. https://doi.org/10.1016/j.nlm.2018.03.021

Taylor, S., Abramowitz, J. S., \& McKay, D. (2012). Non-adherence and non-response in the treatment of anxiety disorders. Journal of Anxiety Disorders, 26, 583-589. https://doi.org/10.1016/j.janxdis.2012.02.010

Wipfli, B., Rethorst, C., \& Landers, D. (2008). The anxiolytic effects of exercise: a meta-analysis of randomized trials and dose-response analysis. Journal of Sport and Exercise Psychology, 30, 392-410. https://doi.org/10.1123/jsep.30.4.392

Wittchen, H. U., Jacobi, F., Rehm, J., Gustavsson, A., Svensson, M., Jönsson, B., . . \& Steinhausen, H. C. (2011). The size and burden of mental disorders and other disorders of the brain in Europe 2010. European Neuropsychopharmacology, 21, 655-679. https://doi.org/10.1016/j.euroneuro.2011.07.018

Wolitzky-Taylor, K. B., Horowitz, J. D., Powers, M. B., \& Telch, M. J. (2008). Psychological approaches in the treatment of specific phobias: a meta-analysis. Clinical Psychology Review, 28, 1021-1037. https://doi.org/10.1016/j.cpr.2008.02.007

Wolpe, J. (1969). Subjective Units of Distress Scale. The Practice of Behavior Therapy.

Yochim, B. P., Mueller, A. E., \& Segal, D. L. (2013). Late life anxiety is associated with decreased memory and executive functioning in community dwelling older adults. Journal of Anxiety Disorders, 27, 567-575. https://doi.org/10.1016/j. janxdis.2012.10.010

Cite this article: Frederiksen KP, Stavestrand SH, Venemyr SK, Sirevåg K, and Hovland A (2021). Physical exercise as an addon treatment to cognitive behavioural therapy for anxiety: a systematic review. Behavioural and Cognitive Psychotherapy 49, 626-640. https://doi.org/10.1017/S1352465821000126 